\title{
Could Mesna and Celery Seed Cotherapy Modulate Oxidative Stress and Inflammation of the Urinary Bladder Induced by Ifosfamide in Rabbits?
}

\begin{abstract}
Ayman M Mousa, (D) ${ }^{1,2}$ Khaled S
Allemailem, (DD ${ }^{1,3}$ Fahad A

Alhumaydhi, (iD ${ }^{3}$ Faris

Alrumaihi, (iD ${ }^{3}$ Ahmad

Almatroudi, (iD ${ }^{3}$ Ameen S

Alwashmi, (iD ${ }^{3}$ Mohammad

Aljasir, (iD ${ }^{3}$ Osamah Al Rugaie, (iD ${ }^{4}$

Abdullah SM Aljohani, (D) ${ }^{5}$ Khaled

EA Soliman, (D) ${ }^{4,6}$ Mohamad $Y$

Rezk, 4,7 Ahmed MS Hegazy, 8

Hanan S Seleem ${ }^{4,10}$

'Department of Basic Health Sciences, College of Applied Medical Sciences, Qassim University, Buraydah, 51452, Saudi Arabia;

${ }^{2}$ Department of Histology and Cell Biology,

Faculty of Medicine, Benha University,

Benha, 13518, Egypt; ${ }^{3}$ Department of

Medical Laboratories, College of Applied

Medical Sciences, Qassim University,

Buraydah, 5I452, Saudi Arabia;

${ }^{4}$ Department of Basic Medical Sciences,

College of Medicine and Medical Sciences,

Qassim University, Unaizah, 5I452, Saudi

Arabia; ${ }^{5}$ Department of Veterinary

Medicine, College of Agricultural and

Veterinary Medicine, Qassim University,

Buraydah, 5I452, Saudi Arabia;

${ }^{6}$ Department of Forensic Medicine and

Clinical Toxicology, Faculty of Medicine,

Sohag University, Sohag, 82524, Egypt;

${ }^{7}$ Department of Medical Physiology, Faculty

of Medicine, Zagazig University, Al-Sharquia,

445 19, Egypt; ${ }^{8}$ Department of Anatomy,

Faculty of Medicine, Northern Border

University, Arar, Saudi Arabia; ${ }^{9}$ Department

of Anatomy and Embryology, Faculty of

Medicine, Benha University, Benha, I3518,

Egypt; ${ }^{10}$ Department of Histology and Cell

Biology, Faculty of Medicine, Menoufia

University, Shebin Elkoum, Egypt
\end{abstract}

Correspondence: Ayman M Mousa

Department of Basic Health Sciences,

College of Applied Medical Sciences, Qassim

University, Buraydah, Saudi Arabia

$\mathrm{Tel}+966$ 59814617l

Email a.mousa@qu.edu.sa
Background: Ifosfamide (IFS) has potential complications such as nephropathy and hemorrhagic cystitis (HC). Although mesna can prevent IFS-induced cystitis by direct binding and neutralization of acrolein, HC symptoms have still been observed clinically in most of these cases. Celery is a powerful healing vegetable that has antioxidant, anti-inflammatory, and anticancer effects. The current study evaluated the synergistic effects of mesna and celery seed on IFS-induced HC in rabbits.

Methods: Twenty male rabbits (four groups) were administered distilled water, IFS, mesna, and mesna+celery seed cotherapy (MCC) for three weeks. The serum and urinary bladder of experimental rabbits underwent biochemical (TNF- $\alpha$, MDA, iNOS, SOD, GPx, and CAT), histopathological and ultrastructural investigations to evaluate the structural changes of the urinary bladder (UB).

Results: IFS injection resulted in severe cystitis with a remarkable increase in the scale of hematuria, elevations of TNF- $\alpha$, MDA, and iNOS activity, and reduced activity of SOD, GPx, and CAT antioxidants. Additionally, the structure of UB exhibited evident mucosal edema and ulceration. In contrast, the MCC regimen group revealed partial synergistic improvement of all mentioned parameters.

Conclusion: IFS induced cystitis by releasing acrolein, which exerted a significant role in the pathogenesis of HC. In contrast, the MCC intake partially ameliorated the UB damage through its antioxidant and anti-inflammatory effects.

Keywords: ifosfamide, mesna, celery, hemorrhagic cystitis, antioxidants, anti-inflammatory

\section{Introduction}

Indeed, chemotherapy regimens usually cause several problems such as allergy and nephropathy. Hemorrhagic cystitis (HC) is a severe complication in patients treated with ifosfamide (IFS), a synthetic analog of cyclophosphamide. ${ }^{1}$ The incidence of $\mathrm{HC}$ in patients treated with high doses of intravenous IFS is $70 \%$, and the mortality rate is $4 \%{ }^{2}$

Patients suffering from HC exhibited various symptoms such as urgency, dysuria, hematuria, and suprapubic pain. Therefore, the complications of HC include hemorrhage, necrosis, fibrosis, and contracture of the urinary bladder (UB). ${ }^{3}$ The pathogenesis of IFS-induced $\mathrm{HC}$ involves direct contact of acrolein with the urothelium, which induces inflammation of the UB. The urothelium is a simple barrier between urine and the UB that gets tremendous importance due to its prominent role in the pathophysiology of UB dysfunction. ${ }^{4}$ Acrolein is a toxic 


\section{Graphical Abstract}

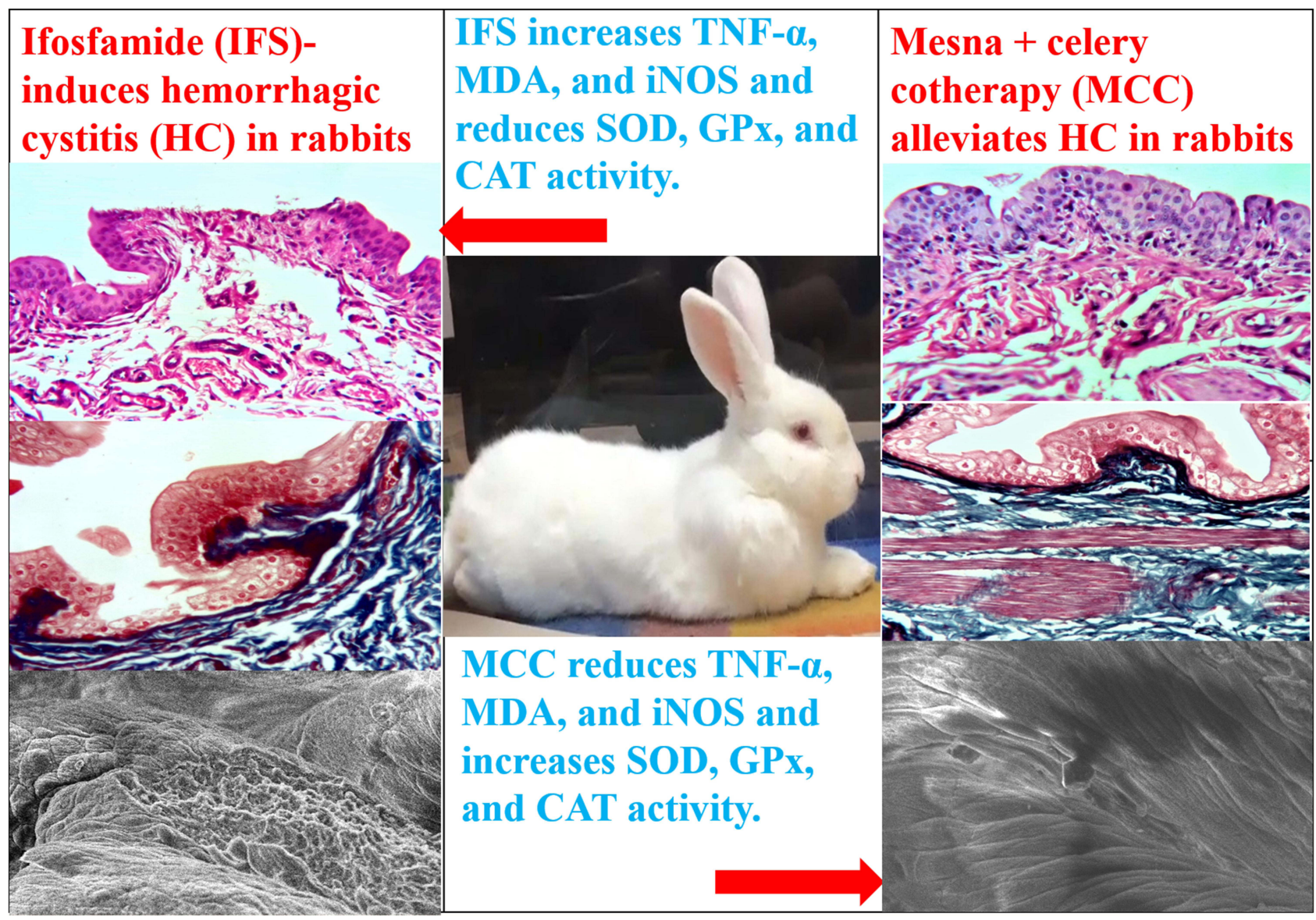

metabolite of IFS formed from hepatic microsomal hydroxylation and excreted by the kidney. Usually, acrolein induces urothelial apoptosis with ulceration and sloughing of the urothelium, followed by damage of the lamina propria and detrusor muscle of the UB. ${ }^{5}$ Some proinflammatory cytokines such as the tumor necrosis factor- $\alpha$ (TNF- $\alpha$ ) and interleukin-1 $\beta$ (IL-1 $\beta$ ) increase the production of nitric oxide (NO) in the cytoplasm of urothelial cells, which plays another significant role in the pathogenesis of HC. ${ }^{6}$ The oxidative stress conditions promote TNF$\alpha$ and NO formation, which induces oxidative stress and inflammation. Malondialdehyde (MDA) is an important oxidative stress biomarker that oxidizes polyunsaturated fatty acids. ${ }^{7}$ Therefore, the UB toxicity mainly arises from reactive nitrogen species (RNS) reaction, including NO and the superoxide $\left(\mathrm{O}_{2}^{-}\right)$. $\mathrm{O} 2$ is abundant in the inflammatory areas and overproduces peroxynitrite ((ONOO), which causes $\mathrm{HC}$ as a final hazardous effect of IFS on the $\mathrm{UB}^{8}{ }^{8}$ Overproduction of the reactive oxygen species (ROS) and RNS during UB inflammation leads to oxidative stress, cellular injury, and necrosis via several mechanisms, including cellular proteins denaturation, DNA damage, and membranes lipid peroxidation. ${ }^{9}$

Mesna (2-mercaptoethanol sulfonate NA) binds directly with acrolein to neutralize it into an inert thioether which passes quickly in urine to the UB without inducing any urothelial damage. Although mesna has been used effectively against IFS-induced cystitis, however; the symptoms of HC persist clinically in about $30 \%$ of cases. ${ }^{10}$ Mesna cannot eradicate IFS-induced $\mathrm{HC}$, which arises from direct contact of the toxic acrolein with the UB mucosa and induces the production of free radicals, RNS and ROS. Therefore, the protective agents against molecular damage in cases of $\mathrm{HC}$ should include various antioxidative enzymatic defense systems such as superoxide dismutase (SOD) and nonenzymatic antioxidants such as celery (Apium graveolens). ${ }^{11}$ 
Celery is a delicious crunchy plant that has a prestigious history as food and medicine. It is one member of the parsley family that originates in the Mediterranean Sea region. Eating raw celery with meals enriches the body with celery nutrient materials, inhibits appetite, reduces body weight (has high contents of water and fibers with few calories), and is a traditional effective remedy for preventing hypertension. ${ }^{12}$ Besides, celery acts as an antioxidant, anti-inflammatory, and diuretic agent to treat arthritis, hepatitis, bronchitis, and asthma. ${ }^{13}$ Celery has a powerful healing effect due to its unique components, such as butylphthalide and flavonoids. ${ }^{14}$ Since celery is safe and cheap, studies focusing on its efficacy as an antioxidant in combination with mesna or other therapeutic modalities against IFS-induced HC are highly required. Therefore, we investigated the effectiveness of mesna and celery cotherapy (MCC) to prevent IFSinduced $\mathrm{HC}$ in the current study.

\section{Materials and Methods}

\section{Animals and Experimental Design}

The current study included an experimental model of adult male New Zealand rabbits to induce HC by IFS administration and preventing it by the $\mathrm{MCC}$ regimen. The rabbits were housed under $23{ }^{\circ} \mathrm{C}$ and fed standard diet ad-libitum. Their age was eight weeks, and their body weight was 1.5 $2.4 \mathrm{~kg}$. The current animal study was accepted by the Research Ethics Committee of Qassim University (Cams1-2019-2-2-I-5467) and was conducted following the ARRIVE guidelines. All procedures were conducted at the animal house of Qassim University under the supervision of a qualified veterinarian (for routine care, general anesthesia, blood sampling, and euthanasia) following the guidelines of Animal Welfare Society, University of London, England. The rabbits were divided into four groups $(n=5)$, which were treated for three weeks. The control group received an intravenous injection (IVI) of $2 \mathrm{~mL}$ distilled water $/ \mathrm{kg} /$ week via the marginal ear vein and the second group (IFS group) received IVI of $45 \mathrm{mg}$ IFS/ $\mathrm{kg} /$ week to induce HC. ${ }^{15}$ In contrast, the third group (IFSM group) received IVI of (45mg IFS $+18 \mathrm{mg}$ mesna/ $\mathrm{kg} /$ week), ${ }^{16}$ and the fourth group (IFSMC group) received IVI of (45 mg IFS+18 mg of mesna/ $\mathrm{kg} /$ week IV) $+15 \mathrm{mg}$ of celery seed/kg/day orally by an orogastric tube. ${ }^{12}$ All rabbits were anesthetized and sacrificed to excise the UB as described before. ${ }^{17}$ The histopathological (HP) and biochemical investigations were conducted to evaluate the efficacy of the MCC regimen on the UB structure in all groups.

\section{Materials}

Ifosfamide vials (holoxan 1gm) and mesna ampoules (uromitexan 400mg) were obtained from Baxter oncology (GmbH, Germany). Celery seed capsules (75 mg) were purchased from Natural factors (Monroe, Canada). The ELISA kits of TNF- $\alpha$ (ABIN6574142), MDA (ABIN775215), inducible nitric oxide synthase (iNOS, ABIN6968940), SOD3 (ABIN6959756), glutathione peroxidase (GPX1, ABIN4969526), and catalase (CAT, ABIN628258) were purchased from the antibodies-online GmbH (Aachen, Germany).

\section{Effects of Mesna and MCC Regimens on} the Scale of Hematuria in IFS-Induced HC Urine dipstick is one of the most valuable and sensitive screening tests for detecting hematuria in unstandardized conditions. It is better than urine microscopy analysis and cytometry techniques because it is more sensitive, accessible, cheaper, and faster than other tools. We collect the urine sample for routine dipstick testing of macroscopic hematuria by putting the rabbit in a cage and confining it with water. Then the rabbit's body was picked up vertically, and its UB was pushed to express some urine every 15 minutes. At the same time, another person held a disposable clean, dry container to catch the stream of urine specimen. The urine dipstick test was immersed into the urine sample to grade the scale of hematuria (from 03 ) in all groups. ${ }^{18,19}$

\section{Biochemical Assessments of Antioxidant and Inflammation Biomarkers}

The rabbit was restrained to avoid inadvertent movement. The dorsal surface of the rabbit's ear tip was shaved, sterilized with $70 \%$ alcohol, and anesthetized locally by EMLA cream 30 minutes before obtaining the blood sample. Two $\mathrm{mL}$ of blood were collected from the central ear vein of each rabbit in a sterile Eppendorf tube using a 20gauge butterfly needle. Blood hemostasis was achieved by pressing the blood sampling site with sterile gauze for 2 minutes. $^{20}$ According to the manufacturer's instructions, the blood samples were centrifuged at $3000 \mathrm{rpm}$ for 10 minutes to obtain serum specimens, stored at $-80^{\circ} \mathrm{C}$ to perform colorimetric assays. The serum activity of SOD3, GPX1, CAT, TNF- $\alpha$, MDA, and iNOS biomarkers was 
measured as described previously ${ }^{21,22}$ to evaluate the efficacy of the MCC regimen.

\section{Effects of Mesna and MCC Regimens on the HP of UB Structure in IFS-Induced $\mathrm{HC}$}

Small specimens from the UB were obtained, processed to get thin sections, and stained with H\&E or Masson trichrome stains to examine the general structure of UB and the extent of HC lesions. The scale of UB damage (ulceration, hemorrhage, and edema) was rated by a pathologist from $0-3$ (no, mild, moderate, and severe changes). ${ }^{23}$ In addition, other parts from the UB were processed and examined by the scanning electron microscope (SEM) as described before to evaluate the extent of UB damage at the mucosal surface. ${ }^{24,25}$

\section{Morphometric Study}

Ten fields from each group were photographed by the CMOS (TC5PRO) digital camera of a light microscope (Jinan, China) at magnification 200X to evaluate the UB structure. Image analysis of UB sections was conducted by Image J V1.50i (NHI/USA) to measure the size of mucosal ulcers and the area percentage of collagen fibers (CF)/10 HPF to evaluate the extent of UB fibrosis.

\section{Statistical Analysis}

The mean (M) \pm standard deviation (SD) of experimental data were statistically analyzed by the SPSS program version 25 (IBM, USA). ANOVA followed by LSD test was conducted to identify the intergroup comparisons (significant values at $P<0.05$ ). The mean $\pm \mathrm{SD}$ of the results were used in the present study because they are not affected by the sample size and indicate how accurately the mean represents the sample of data. At first, the normality tests (skewness and Kurtosis) were performed and revealed the normal data distribution.

\section{Results}

\section{Mesna and MCC Effects on the Scale of Hematuria of IFS-Induced HC}

Measurement of the scale of hematuria by the urine dipstick test in Figure 1 revealed significant increases in the scale of hematuria in the IFS group compared to the control and IFSMC groups.

\section{Mesna and MCC Effects on the Antioxidant Enzymes of IFS-Induced HC}

Quantitative analysis of the antioxidant enzymes in Figure 2 revealed significant reductions in the serum activity of SOD, GPx, and CAT in the IFS group

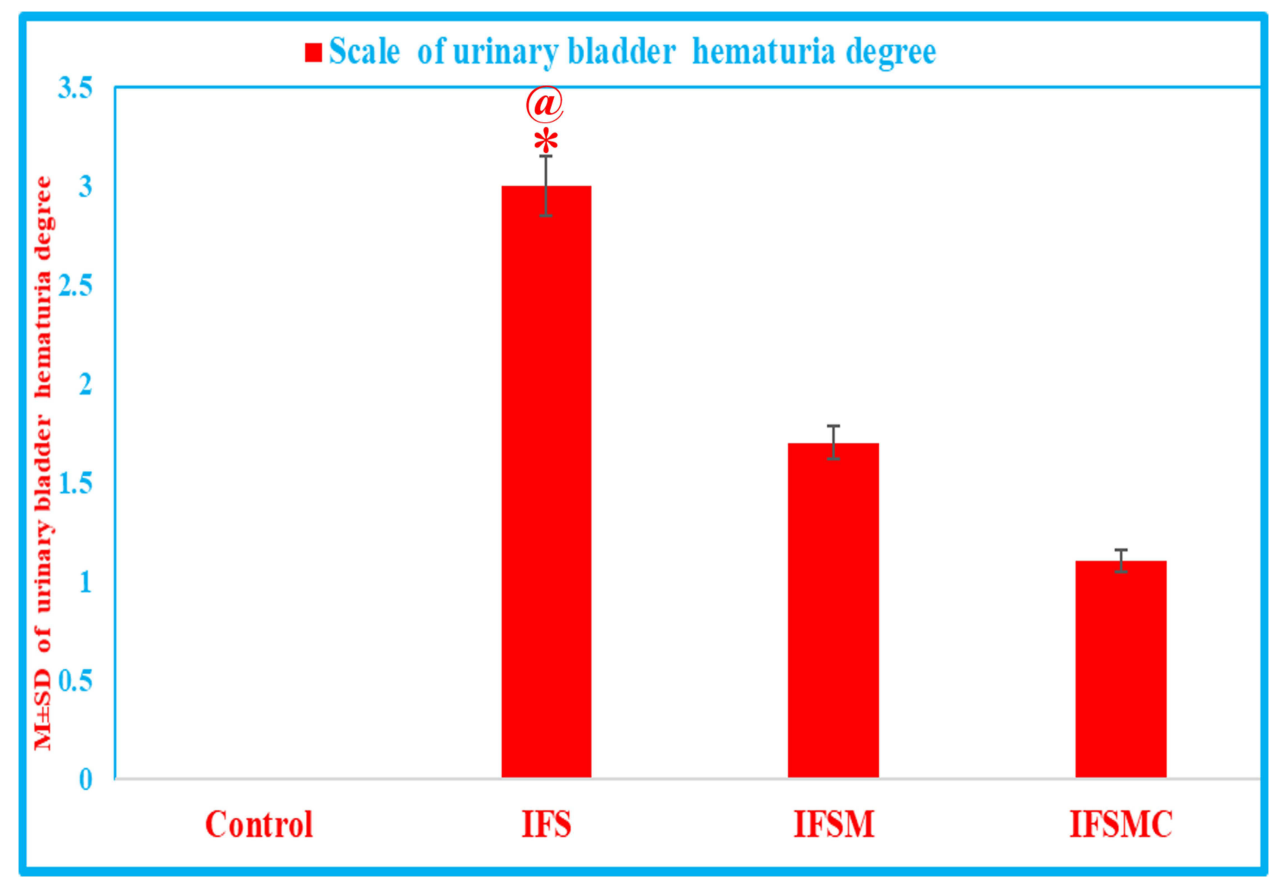

Figure I Statistical analysis of mesna and MCC effects on the scale of hematuria in IFS-induced HC. The IFS group shows significant increases in the scale of hematuria compared to the control and IFSMC groups. $* \mathrm{P}<0.05$ vs the control and ${ }^{@} \mathrm{P}<0.05$ vs the IFSMC group. 


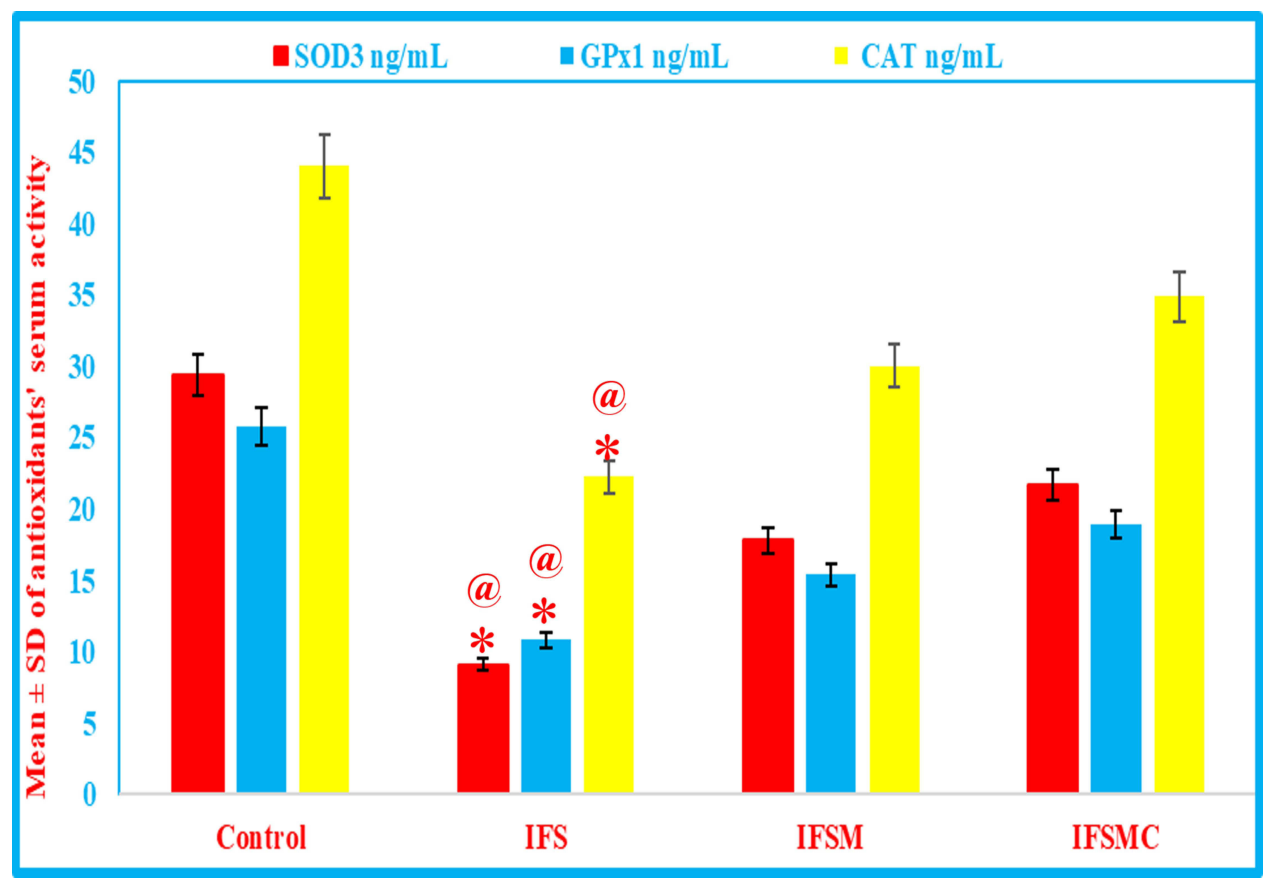

Figure 2 Statistical analysis of mesna and MCC effects on the antioxidants' serum activity of IFS-induced HC. The SOD3, GPxI, and CAT activity show significant reductions in the IFS group compared to the control and IFSMC groups. ${ }^{*} \mathrm{P}<0.05$ vs the control and ${ }^{@} \mathrm{P}<0.05$ vs the IFSMC group.

compared to the control and IFSMC groups, indicating the marked oxidative stress effects of IFS on the IFS group. In contrast, administration of $\mathrm{MCC}$ in the IFSMC group resulted in significant elevations in all antioxidants, which attenuated the oxidative stress and $\mathrm{HC}$ in the IFSMC group.

\section{Mesna and MCC Effects on the Inflammatory Biomarkers of IFS-Induced $\mathrm{HC}$}

Quantitative analysis of ELISA results in Figure 3 revealed significant increases in the TNF- $\alpha, \mathrm{MDA}$, and iNOS serum activity of the IFS group compared to their normal activity in the control and IFSMC groups, indicating the marked inflammatory effects of IFS on the IFS group. In contrast, administration of MCC in the IFSMC group resulted in a remarkable reduction in the activity of all inflammatory biomarkers, indicating that MCC attenuated $\mathrm{HC}$ in the IFSMC group due to the reduction of oxidative stress and inflammatory mediators' biomarkers.

\section{Mesna and MCC Effects on the HP of UB Structure in IFS-Induced HC}

Figure 4 revealed typical UB structure in the control and IFSMC groups, indicating the protective effects of the
MCC regimen against $\mathrm{HC}$ in the IFSMC group. In contrast, an evident abnormality had been encountered in the structure of UB (urothelial ulceration and mucosal sloughing) in the IFS group, compared to the control group, indicating the harmful effects of IFS. In addition, the IFSM group revealed mild ulceration of the UT compared to the IFS group, indicating the partial protection of mesna monotherapy against IFS-induced urotoxicity. Statistical analysis of the ulcers' size confirmed the induction of $\mathrm{HC}$ in the IFS group compared to the control group and the evident protection of UB by the MCC regimen in the IFSMC group compared to the IFSM group.

\section{Mesna and MCC Effects on UB CF Deposition in the IFS-Induced $\mathrm{HC}$}

Figure 5 exhibited excessive $\mathrm{CF}$ deposition in the lamina propria of UB, indicating fibrosis of the UB wall in the IFS group. In contrast, the UB of the IFSM \& IFSMC groups revealed mild deposition of $\mathrm{CF}$.

\section{Mesna and MCC Effects on the UB Ultrastructure of IFS-Induced HC}

Figure 6 revealed the ultrastructure of UB mucosa by the SEM. The UB of the control and IFSMC groups revealed normal UB transitional epithelium (TE), indicating a remarkable preventive effect of the $\mathrm{MCC}$ regimen 


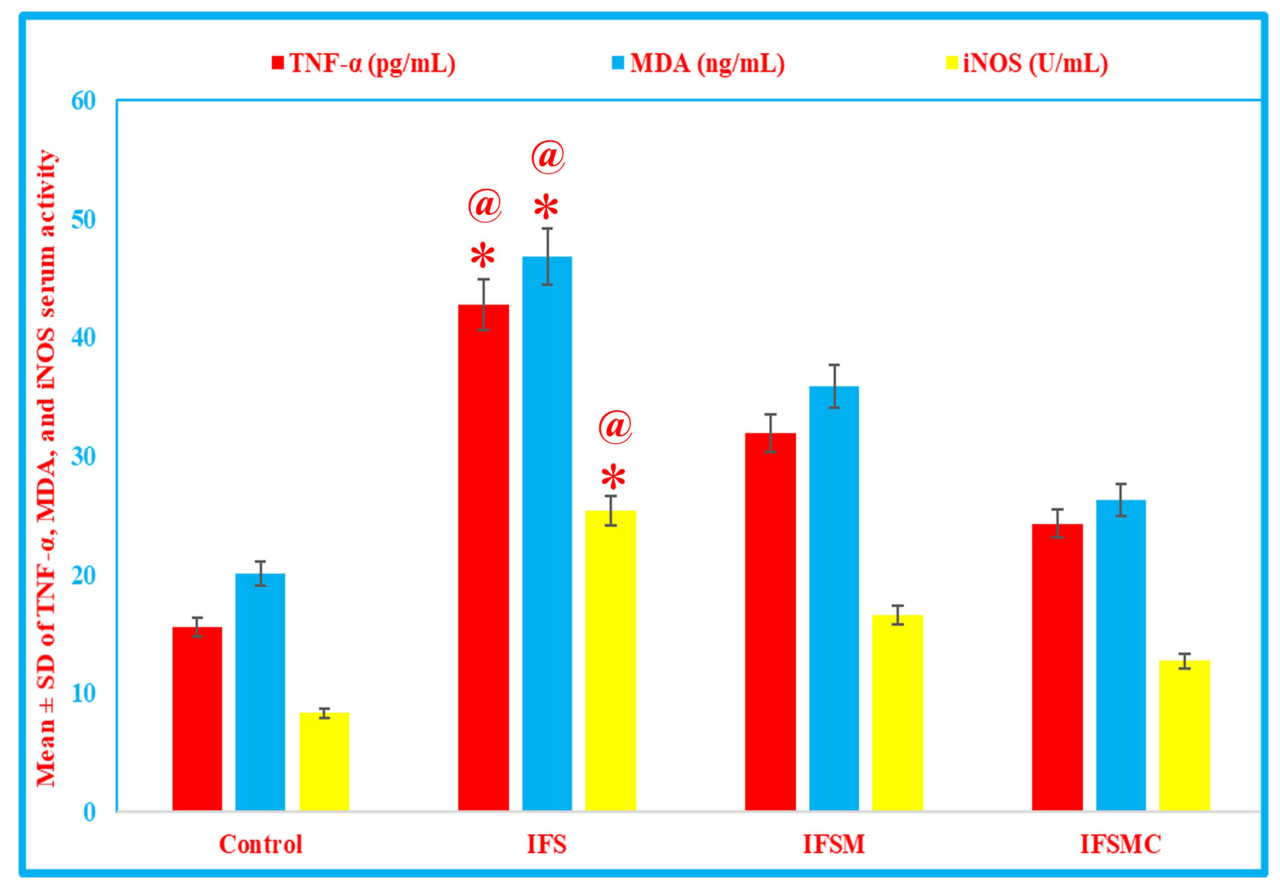

Figure 3 Statistical analysis of mesna and MCC effects on the inflammatory biomarkers of IFS-induced HC. The TNF- $\alpha$, MDA, and iNOS activity show significant increases in the IFS group compared to the control and IFSMC groups. ${ }^{*} \mathrm{P}<0.05$ vs the control and ${ }^{\circledR} \mathrm{P}<0.05$ vs the IFSMC group.

against the $\mathrm{HC}$ in the IFSMC group. In contrast, the IFS group exhibited a significant increase in the area percentage of mucosal ulcers, indicating the hazardous effects of IFS on the UB of the IFS group. At the same time, the area percentage of mucosal ulcers was minimally reduced in the IFSM group, indicating inadequate protection of the UB by mesna monotherapy in the IFSM group. Statistical analysis of the area percentage of mucosal ulcers confirmed the existence of $\mathrm{HC}$ in the IFS group compared to the control and IFSMC groups.

\section{Discussion}

Today, it is evident that IFS-induced HC mainly arises from inflammation and damage of the UB urothelium due to elevated ROS and proinflammatory cytokines. Unfortunately, no single "magic bullet" is sufficient for the treatment of $\mathrm{HC}$, and the need to introduce other promising regimens for the prevention of $\mathrm{HC}$ is of prime interest. Thus, combined treatment modalities such as mesna with natural herbs such as celery seeds are highly recommended, and the anti-inflammatory agents are expected to be effective for the treatment of HC. This type of cotherapy has remarkable antioxidants and antiinflammatory effects. ${ }^{26}$

In the current study, we undertook bladder samples from pretreated rabbits with IFS, mesna, and celery seeds to provide insights into the protective mechanisms of the MCC regimen and to evaluate the upregulation of proinflammatory cytokines in cases of HC. The degree of hematuria in Figure 1 and the serum levels of TNF- $\alpha$, MDA, and iNOS (proinflammatory and lipid peroxidation cytokines) were elevated in the IFS group and significantly reduced in the IFSMC group by coadministration of mesna and CES in Figure 3. Additionally, the serum levels of SOD3, GPX1, and CAT significantly reduced in the IFS group compared to the control and IFSMC groups in Figure 2. In contrast, administration of MCC in the IFSMC group resulted in significant reductions in the degree of hematuria and the levels of TNF- $\alpha$, MDA, and iNOS, with significant elevations in the SOD3, GPX1, and CAT levels, indicating that the $\mathrm{MCC}$ regimen reduced the oxidative stress in the IFSMC group, which attenuated the degree of HC. Moreover, the main HP features of HC in Figures 4-6 were urothelial damage and necrosis in the IFS group, which were significantly reduced in the IFSMC group more than in the IFSM group.

The induction of HC after IFS injection could be explained by numerous studies, which reported that direct contact of acrolein with the UB urothelium and increased production of the ROS and RNS compounds are the leading causes of $\mathrm{HC}^{27,28}$ Korkmaz et al mentioned that acrolein reacts with various proteins, DNA, and 


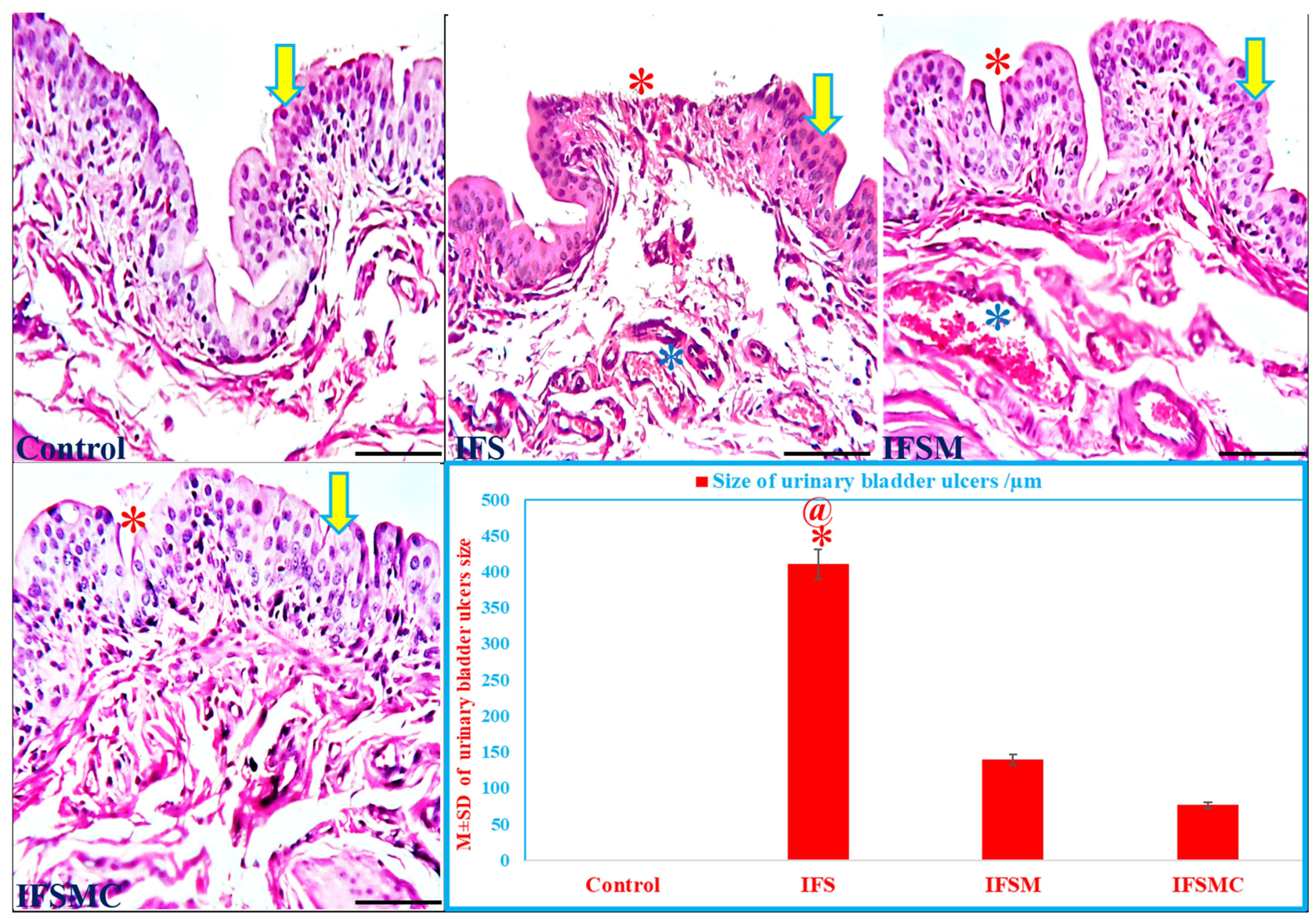

Figure 4 Mesna and MCC effects on the structure of urinary bladder (UB) of IFS-induced HC. The UB of the control and IFSMC groups exhibit the normal structure of the transitional epithelium (yellow arrow). In contrast, the UB of the IFS group reveals a large mucosal ulcer (red *) and congested blood vessels (blue *). At the same time, the IFSM group exhibits a mild improvement of the UB structure with a small ulcer (red *) compared to the IFS group. H\&E; 200x, bar $=100 \mu \mathrm{m}$. Statistical analysis of the size of UB mucosal ulcers. ${ }^{*} \mathrm{P}<0.05$ vs the control and ${ }^{@} \mathrm{P}<0.05$ vs the IFSMC group.

glutathione, causing cellular necrosis and depletion. At the same time, the pathogenesis of $\mathrm{HC}$ is influenced by the toxic effects of acrolein and the cascade of cellular inflammatory reactions on the urothelium of UB, with consequent injury of these tissues, which are evoked by the release of numerous proinflammatory mediators. ${ }^{29}$ Moreover, acrolein activates the inflammatory mediators such as the nuclear factor-kB (NF-kB) and TNF- $\alpha$, which initiate lipids peroxidation and exaggerate oxidative stress. After entering the UB, acrolein increases the production of ROS in the urothelium, reacts with glutathione and aldehyde dehydrogenase to produce the toxic acrolein metabolites, which are responsible for the overproduction of free oxygen radicals by the urothelium. ${ }^{30}$ In addition, NO and peroxynitrite (ONOO) activation impairs the urothelial integrity and increases the severity of UB damage. NO is a biological molecule, which is produced in high concentrations and competes with the endogenous SOD. The inflammatory cells such as macrophages potentially release NO and superoxide, leading to overproduction of ONOO via iNOS activation, which is an essential factor in the process of tissue damage. ${ }^{31}$ Mesna is rapidly oxidized in the circulation into an inactive dimer (dimesna), which is excreted in urine and creates a nontoxic compound in the UB by direct combination with the double bonds of acrolein. ${ }^{32}$ Although mesna is effective in protection against $70 \%$ of $\mathrm{HC}$ cases (due to its limited antioxidant activity); however, it needs coadministration of other synergistic antioxidant agents to support its weak protective effects. Fortunately, a recent study reported that coadministration of mesna with antioxidant agents has been widely accepted as a novel chemoprotective regimen and could be effective in preventing UB inflammatory conditions as they exhibit remarkable protective effects and almost abolish damage of the UB. ${ }^{33}$

Dietary modifications have been incorporated as the first line of treatment against HC. Numerous literature has proved that herbal medicine plants such as celery are 


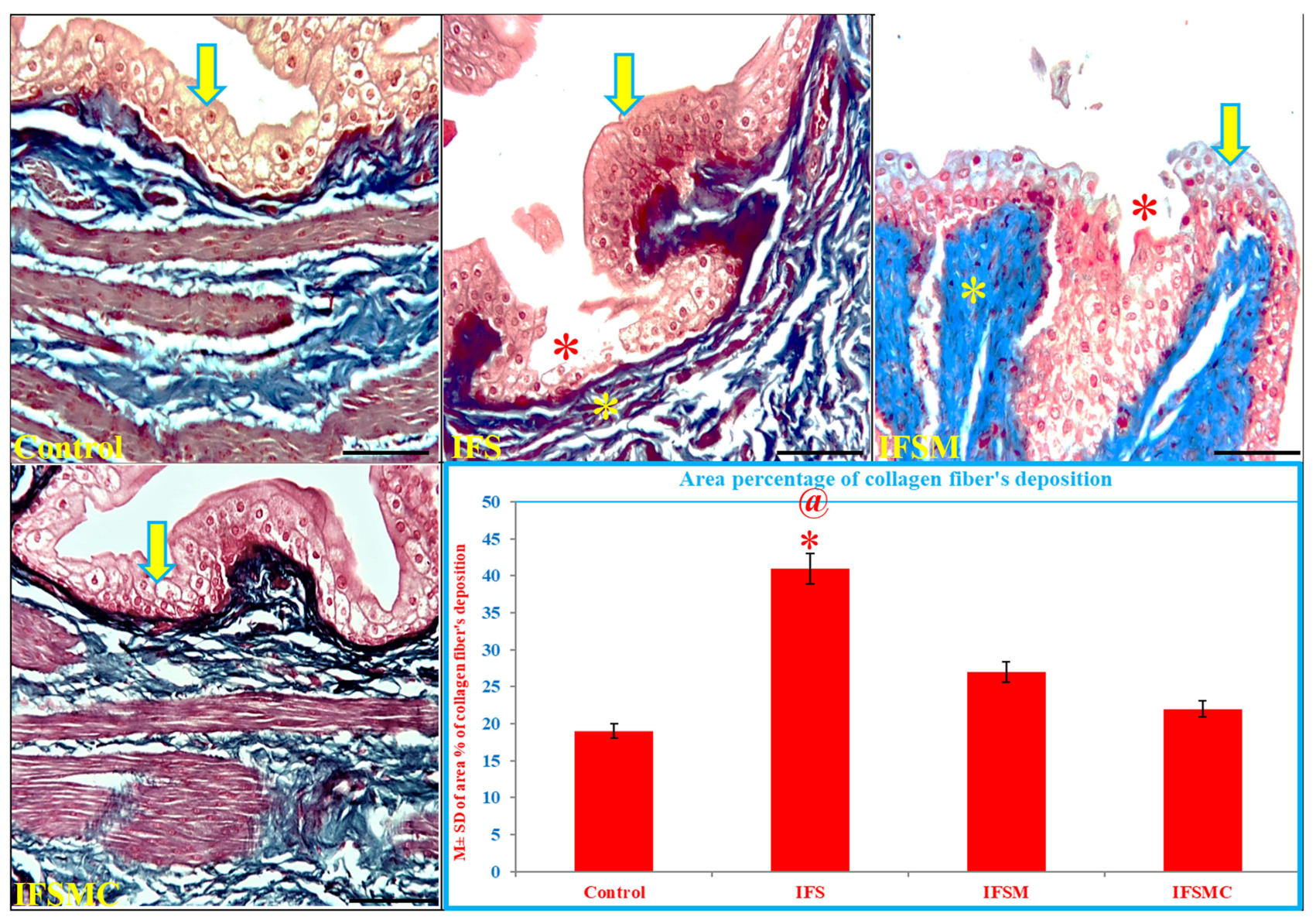

Figure 5 Mesna and MCC effects on UB collagen fibers deposition of IFS-induced HC. The UB of the control and IFSMC groups exhibit normal transitional epithelium (yellow arrow) over the lamina propria. In contrast, the UB shows obvious large mucosal ulcer (red *) and collagen fibers deposition (yellow *) in the IFS group with moderate improvements of fibrosis in the IFSM group. Masson's trichrome; 200x, bar $=100 \mu \mathrm{m}$. Statistical analysis of collagen fibers' deposition in the UB of all groups. $* \mathrm{P}<0.05$ vs the control and ${ }^{@} \mathrm{P}<0.05$ vs the IFSMC group.

safe, cheap, and could be used effectively to prevent numerous urinary tract diseases such as HC. Hence, the non-enzyme antioxidants, such as celery and ginseng, are crucial elements in reducing molecular damage, enhancing the antioxidative defense system with decreased blood urea nitrogen and serum creatinine levels in numerous diseases. ${ }^{34}$ Therefore, in the current study, we examined whether the MCC regimen shows better results than mesna monotherapy in IFS-induced HC or not. In the present study, this hypothesis has been supported by improving biochemical and histological results, which may reduce ROS and RNS activity and the proinflammatory cytokines in the IFSMC group. The most active constituents of celery seeds are d-limonene (60\%), terpenes, and selinene, which can enhance the activity of depleted antioxidant enzymes, including SOD, GPx, and CAT enzymes. ${ }^{35,36} \mathrm{In}$ addition, the novel chemoprotective regimen MCC ameliorated the damage of UB through modulating the antioxidant status, enhancing the scavenging activity of free radicals (ROS and RNS), and regulating the proinflammatory cytokines. ${ }^{37}$ At the same time, the celery seeds extensively decreased the MDA, iNOS, and ONOO markers and reduced the lipids peroxidation activity. ${ }^{38}$ Furthermore, celery seeds have proved anti-inflammatory properties (decreased levels of IL-1, IL-6, and TNF- $\alpha$ ), which may contribute to better efficacy of IFS therapy and prevent IFS-induced HC and urotoxicity. ${ }^{39,40}$

\section{Conclusion}

To sum up, the development of $\mathrm{HC}$ is a severe side effect that remarkably limits the use of IFS as an antineoplastic agent. Based on the current results, the protective MCC regimen produced lesser inflammation, ulceration, and hemorrhage of the UB and exhibited apparent restoration of the antioxidant enzymes to their normal activity. In addition, the IFSMC group alleviated the symptoms of $\mathrm{HC}$ and revealed a significant reduction of TNF- $\alpha$, MDA, and iNOS activity in the IFSMC group compared to the UB 


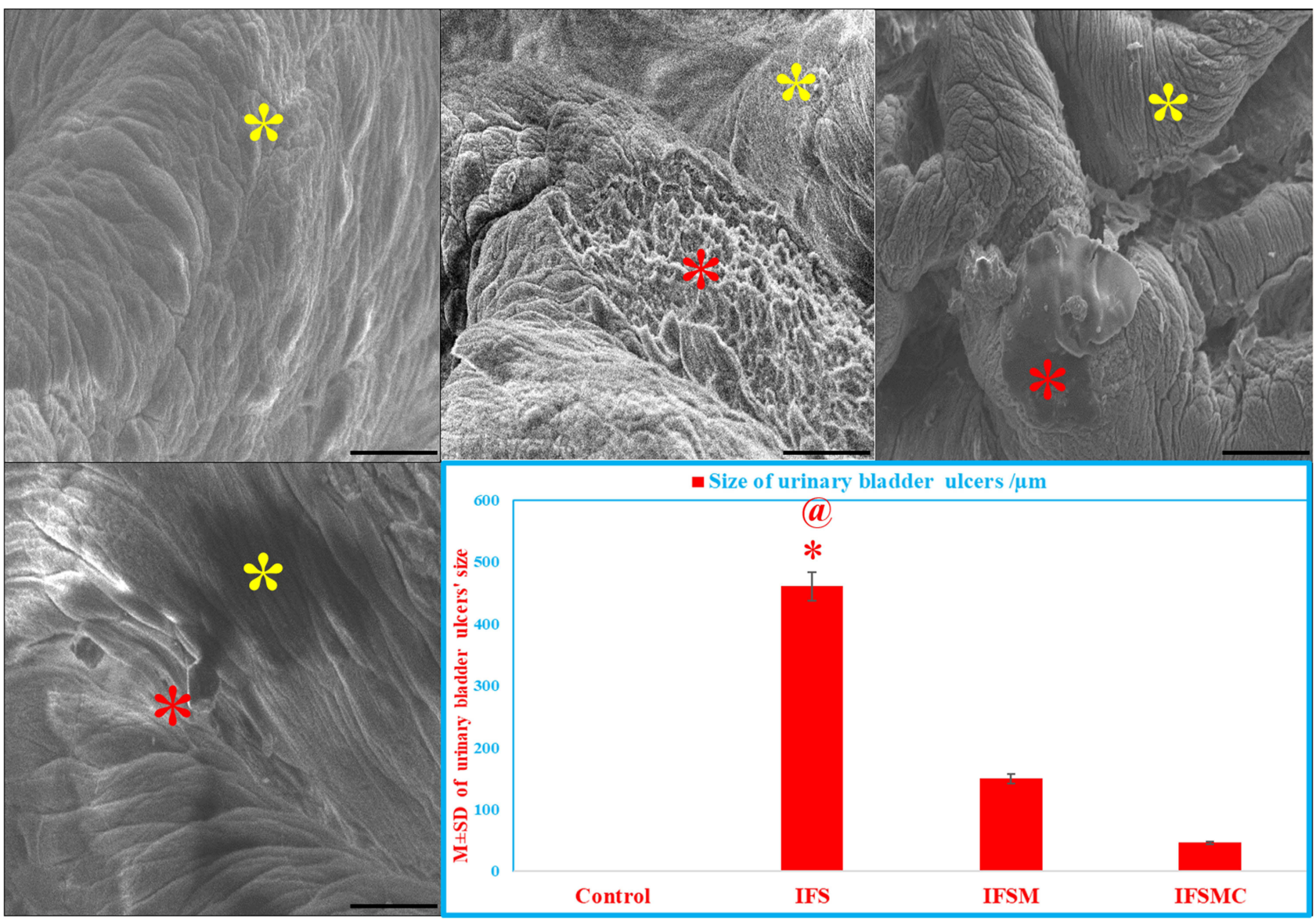

Figure 6 Mesna and MCC effects on the UB ultrastructure of IFS-induced HC. The control and IFSMC groups reveal normal UB surface transitional epithelium (yellow *). In contrast, the IFS group shows significant increases in the area percentage of ulcerated mucosa (red *), which is minimally reduced in the IFSM group. SEM; $700 x$, bar $=30 \mu m$. Statistical analysis of the area percentage of UB ulcers in all groups. $* \mathrm{P}<0.05$ vs the control and ${ }^{@} \mathrm{P}<0.05$ vs the IFSMC group.

of IFS-treated rabbits. Therefore, the MCC regimen seems to be a novel promising chemoprotection cotherapy, which could control the symptoms of IFS-induced HC. However, the improvement of UB histology and the antioxidants activity in cases of IFS-induced HC by the MCC regimen did not ensure full recovery of UB toxicity. This finding deserves the need for further experimental explorations focusing on the efficacy of other celery therapeutic modalities, such as combining celery with honey against induced HC. Future studies may find new chemoprotection agents that can provide a total reduction of IFS-evoked HC.

\section{Data Sharing Statement}

All relevant data have been provided in the manuscript.

\section{Funding}

The authors gratefully acknowledge Qassim University, represented by the Deanship of Scientific Research, on the financial support for this research under the number
[Cams1-2019-2-2-I-5467] during the academic year 1440 $\mathrm{AH} / 2019$ AD.

\section{Disclosure}

The authors declare no conflicts of interest in this study. The funder had no role in the design, data collection, analyses, or interpretation of the study, writing the manuscript, or publishing the results.

\section{References}

1. Matz EL, Hsieh MH. Review of advances in uroprotective agents for cyclophosphamide- and ifosfamide-induced hemorrhagic cystitis. Urology. 2017;100:16-19. doi:10.1016/j.urology.2016.07.030

2. Hamsa TP, Kuttan G. Protective role of Ipomoea obscura (L.) on cyclophosphamide-induced uro- And nephrotoxicities by modulating antioxidant status and pro-inflammatory cytokine levels. Inflammopharmacology. 2011;19(3):155-167. doi:10.1007/s10787010-0055-3

3. Huang CJ, Lee FK, Chen SK, Chien CC, Wu ST, Wang YC. Clinical significance of interleukin- 6 and inducible nitric oxide synthase in ketamine-induced cystitis. Int J Mol Med. 2018;41(2):836-844. doi:10.3892/ijmm.2017.3264 
4. Bury MI, Fuller NJ, Meisner JW, et al. The promotion of functional urinary bladder regeneration using anti-inflammatory nanofibers. Biomaterials. 2014;35(34):9311-9321. doi:10.1016/j.biomaterials.20 14.07.057

5. Haldar S, Dru C, Bhowmick NA. Mechanisms of hemorrhagic cystitis. Am j Clin Exp Urol. 2014;2(3):199-208.

6. Engin S, Barut EN, Barut B, Duman MK, Kaya C, Kerimoglu G. Uroprotective effect of pantoprazole against cyclophosphamide-induced cystitis in mice. Supportive Care Cancer. 2019;27(11):4273-4281. doi:10.1007/s00520-019-04731-2

7. Mbanefo EC, Le L, Zee R, et al. IPSE, a urogenital parasite-derived immunomodulatory protein, ameliorates ifosfamide-induced hemorrhagic cystitis through downregulation of pro-inflammatory pathways. Sci Rep. 2019;9(1):1-13. doi:10.1038/s41598-018-38274-Z

8. Sherif IO, Nakshabandi ZM, Mohamed MA, Sarhan OM. Uroprotective effect of oleuropein in a rat model of hemorrhagic cystitis. Int $J$ Biochem Cell Biol. 2016;74:12-17. doi:10.1016/j. biocel.2016.02.012

9. Shimada K, Fujii T, Anai S, Fujimoto K, Konishi N. ROS generation via NOX4 and its utility in the cytological diagnosis of urothelial carcinoma of the urinary bladder. BMC Urol. 2011;11. doi:10.1186/ 1471-2490-11-22

10. Filiz S, Enis U, Mustafa I, Aysegul C, Elvan B. Protective effect of mesna on intestinal ischemia-reperfusion injury by nitric oxide and arginase in an experimental rat model. Int $J$ Pharmacol. 2017;13 (8):1038-1046. doi:10.3923/ijp.2017.1038.1046

11. Sadir S, Deveci S, Korkmaz A, Oter S. Alpha-tocopherol, beta-carotene and melatonin administration protects cyclophosphamide-induced oxidative damage to bladder tissue in rats. Cell Biochem Funct. 2007;25(5):521-526. doi:10.1002/cbf.1347

12. Brankovi S, Radenkovi M, Veljkovi S, Kosti M, Miladinovi B, Pavlovi D. Hypotensive and cardioinhibotory effects of the aqueous and ethanol extracts of celery (apium graveolens, apiaceae). Acta Med Medianae. 2010;49(1):13-16.

13. Khanum F, Anand T, Ilaiyaraja N, et al. Health Food for Soldiers. Defence Life Sci J. 2017;2(2):111. doi:10.14429/dlsj.2.11363

14. Fazal S, Singla R. Review on the pharmacognostical \& pharmacological characterization of apium graveolens linn. Indo Global J Pharm Sci. 2012;2(1):36-42.

15. Ypsilantis P, Papaioannou N, Psalla D, Politou M, Simopoulos C. Effects of single dose administration of ifosfamide on testes and semen characteristics in the rabbit. Reprod Toxicol. 2003;17 (2):237-245. doi:10.1016/S0890-6238(02)00127-2

16. Ypsilantis P, Tentes I, Assimakopoulos SF, Kortsaris A, Scopa CD, Simopoulos C. Mesna ameliorates intestinal mucosa damage after ifosfamide administration in the rabbit at a dose-related manner. J Surg Res. 2004;121(1):84-91. doi:10.1016/j.jss.2004.03.003

17. Mousa AM, El-Mahalaway AM, El-Desoky RE. The protective effects of sodium hyaluronate on corneal injuries induced by chlorinated water in adult male rabbits: a histological and immunohistochemical study. Egypt J Histol. 2018;41(2):140-149. doi:10.21608/ EJH.2018.13835

18. Bataille A, Wetzstein M, Hertig A, Vimont S, Rondeau E, Galichon P. Evidence of dipstick superiority over urine microscopy analysis for detection of hematuria. BMC Res Notes. 2016;9(1):9-11. doi:10.1186/s13104-016-2240-y

19. Diviney J, Jaswon MS. Urine collection methods and dipstick testing in non-toilet-trained children. Pediatr Nephrol. 2021;36(7):16 97-1708. doi:10.1007/s00467-020-04742-w

20. Moore DM, Zimmerman K, Smith SA. Hematological assessment in pet rabbits: blood sample collection and blood cell identification. Clin Lab Med. 2015;35(3):617-627. doi:10.1016/j.cll.2015.05.010

21. Hussien NI, Mousa AM. Could nitric oxide be a mediator of action of oxytocin on myocardial injury in rats? (Biochemical, histological and immunohistochemical study). Gen Physiol Biophys. 2016;35 (03):353-362. doi:10.4149/gpb_2015049
22. Bitencourt MR, Patias LD, Beck M, et al. Evaluation of the biochemical, inflammatory and oxidative profile of obese patients given clinical treatment and bariatric surgery. Clin Chim Acta. 2017;465:72-79. doi:10.1016/j.cca.2016.12.012

23. Mousa AM, Almatroudi A, Alwashmi AS, et al. Thyme oil alleviates Ova-induced bronchial asthma through modulating Th2 cytokines, IgE, TSLP and ROS. Biomed Pharmacother. 2021;140(8):111726. doi:10.1016/j.biopha.2021.111726

24. Poveda-Pagán EJ, Hernández-Sánchez S, Rhys-Jones-López L, Palazón-Bru A, Lozano-Quijada C. Scanning electron microscopy examination of needle tips after different procedures of deep dry needling in humans. Sci Rep. 2018;8(1):1-10. doi:10.1038/s41598018-36417-w

25. Mousa AM, Aldebasi YH. L-carnosine mitigates interleukin- $1 \alpha$ induced dry eye disease in rabbits via its antioxidant, antiinflammatory, antiapoptotic, and antifibrotic effects. Cutan Ocul Toxicol. 2021;40(3):241-251. doi:10.1080/15569527.2021.1935995

26. Ozcan A, Korkmaz A, Oter S, Coskun O. Contribution of flavonoid antioxidants to the preventive effect of mesna in cyclophosphamide-induced cystitis in rats. Arch Toxicol. 2005;79 (8):461-465. doi:10.1007/s00204-005-0647-7

27. Batista CKLP, Brito GAC, Souza MLP, Leitão BTA, Cunha FQ, Ribeiro RA. A model of hemorrhagic cystitis induced with acrolein in mice. Braz J Med Biol Res. 2006;39(11):1475-1481. doi:10.1590/ S0100-879X2006001100011

28. Vieira MM, Castro Brito GA, Belarmino-Filho JN, et al. Use of dexamethasone with mesna for the prevention of ifosfamide-induced hemorrhagic cystitis. Int $J$ Urol. 2003;10 (11):595-602. doi:10.1046/j.1442-2042.2003.00709.x

29. Sherif IO. Uroprotective mechanisms of natural products against cyclophosphamide-induced urinary bladder toxicity: a comprehensive review. Acta Sci Polonorum, Technologia Aliment. 2020;19 (3):333-346. doi:10.17306/J.AFS.0832

30. Korkmaz A, Topal T, Oter S. Pathophysiological aspects of cyclophosphamide and ifosfamide induced hemorrhagic cystitis; Implication of reactive oxygen and nitrogen species as well as PARP activation. Cell Biol Toxicol. 2007;23(5):303-312. doi:10.1007/s10565-006-0078-0

31. Korkmaz A, Oter S, Sadir S, et al. Peroxynitrite may be involved in bladder damage caused by cyclophosphamide in rats. $J$ Urol. 2005;173(5):1793-1796. doi:10.1097/01.ju.0000154344.80669.e3

32. Barut EN, Engin S, Barut B, et al. Uroprotective effect of ambroxol in cyclophosphamide-induced cystitis in mice. Int Urol Nephrol. 2019;51(5):803-810. doi:10.1007/s11255-019-02128-y

33. Abd El-Baset SA, Abd El-Haleem MR, Abdul-Maksoud RS, Kattaia AAA. Mesna ameliorates acute lung injury induced by intestinal ischemia-reperfusion in rats. Sci Rep. 2021;11(1):13356. doi:10.1038/s41598-021-92653-7

34. Wongtawatchai T, Sarsutham K, Sukketsiri W, Tipmanee V, Chonpathompikunlert P. Anti-stress effects of Apium graveolens on rats subjected to immobilization. Int Food Res J. 2017;24 (4):1490-1499.

35. Kooti W, Daraei N. A review of the antioxidant activity of celery (Apium graveolens L). J Evid Based Complementary Altern Med. 2017;22(4):1029-1034. doi:10.1177/2156587217717415

36. Dąbrowska JA, Kunicka-Styczyńska AŚK. Biological, chemical, and aroma profiles of essential oil from waste celery seeds (Apium graveolens L.). J Essent Oil Res. 2020;32(4):308-315. doi:10.1080/ 10412905.2020.1754937

37. Sukketsiri W, Chonpathompikunlert P, Tanasawet S, Choosri N, Wongtawatchai T. Effects of Apium graveolens extract on the oxidative stress in the liver of adjuvant-induced arthritic rats. Preventive Nutr Food Sci. 2016;21(2):79-84. doi:10.3746/pnf.2016.21.2.79

38. Wang S, Ma F, Huang L, et al. D1-3-n-Butylphthalide (NBP): a promising therapeutic agent for ischemic stroke. CNS Neurol Disord - Drug Targets. 2018;17(5):338-347. doi:10.2174/187152731 7666180612125843 
39. Li MY, Feng K, Hou XL, et al. The genome sequence of celery (Apium graveolens L.), an important leaf vegetable crop rich in apigenin in the Apiaceae family. Hortic Res. 2020;7(1). doi:10. 1038/s41438-019-0235-2
40. Sung B, Chung HY, Kim ND. Role of apigenin in cancer prevention via the induction of apoptosis and autophagy. J Cancer Prev. 2016;21 (4):216-226. doi:10.15430/jcp.2016.21.4.216

\section{Publish your work in this journal}

The Journal of Inflammation Research is an international, peerreviewed open-access journal that welcomes laboratory and clinical findings on the molecular basis, cell biology and pharmacology of inflammation including original research, reviews, symposium reports, hypothesis formation and commentaries on: acute/chronic inflammation; mediators of inflammation; cellular processes; molecular mechanisms; pharmacology and novel anti-inflammatory drugs; clinical conditions involving inflammation. The manuscript management system is completely online and includes a very quick and fair peerreview system. Visit http://www.dovepress.com/testimonials.php to read real quotes from published authors.

Submit your manuscript here: https://www.dovepress.com/journal-of-inflammation-research-journal 